\title{
LA ORIENTACIÒN EDUCATIVA PARA EL DESARROLLO DE NOCIONES ELEMENTALES DE MATEMÀTICA EN EL NIÑO SORDO
}

\section{EDUCATIONAL GUIDANCE FOR THE DEVELOPMENT OF ELEMENTARY NOTIONS OF MATHEMATICS IN DEAF CHILDREN}

\author{
M Sc. Rosamaría González Cuesta ${ }^{1}$ \\ Universidad de Matanzas, Cuba.
}

\section{RESUMEN}

Se aborda un tema actual en la educación cubana de los niños sordos de la primera infancia, relacionado con la preparación de los contextos en prácticas educativas inclusivas que garanticen una educación de calidad. Se analiza el desarrollo del proceso educativo inclusivo y las transformaciones en la educación de sordos, en su continuo perfeccionamiento a partir del modelo educativo bilingüe cubano. Las sugerencias propuestas dirigidas a mejorar el desempeñode la familia y otros agentes educativos para el desarrollo de nociones de matemática en el

$1 \quad$ Profesora Auxiliar de la carrera PedagogíaPsicología. Máster en Ciencias de la educación superior. Jefa de la disciplina Fundamentos fisiológicos y psicológicos del aprendizaje en el Departamento docente Pedagogía Psicología de la Facultad de Educación. Universidad de Matanzas. https://orcid.org/0000-0002-2949-2307 . afiliada de Redipe. Capítulo Cuba. Matanzas. rosa.cuesta@umcc.cu niño sordo, da respuesta a la necesidad de potenciar el desarrollo de diferentes áreas de la actividad psíquica del menor para contribuir al desarrollo de los procesos del pensamiento y su preparación para su ingreso a la escuela.

PALABRAS CLAVE: primera infancia, sordos, orientación educativa, nociones elementales de matemática.

It discusses a current issue in Cuban education of deaf children in early childhood, related to the preparation of contexts in inclusive educational practices that ensure quality education. It analyzes the development of the inclusive educational process and the transformations in the education of the deaf, in their continuous improvement based on the Cuban bilingual educational model. The proposed suggestions aimed at improving the performance of the 
family and other educational agents for the development of notions of mathematics in the deaf child, responds to the need to enhance the development of different areas of psychic activity of the child to contribute to the development of thought processes and their preparation for entry into school.

Keywords: early childhood, deafness, educational guidance, elementary notions of mathematics.

\section{INTRODUCCIÓN}

El papel de la educación y las influencias sociales resulta decisivo en el desarrollo de cada persona, es por eso que el desarrollo de la labor de orientación a los educadores, la familia y la comunidad es una necesidad en la sociedad cubana actual, donde se crean todas las condiciones para hacer realidad la inclusión educativa.

Desde una educación con un enfoque optimista y desarrollador, se concibe que todos los niños sin excepción pueden tener éxito en su desarrollo integral, si se crean las condiciones necesarias para dar una respuesta oportuna a sus necesidades educativas desde la primera infancia.

La familia, como primera agencia educativa que interviene en la formación y desarrollo de la personalidad de su descendencia desde el nacimiento de los hijos, cumple funciones importantísimas; en el caso de los niños sordos la comunicación ocupa una posición principal, debido a la situación bilingüe particular en la que transcurre la convivencia social de estos niños.

Es por ello que la preparación de la familia para que cumpla con esta importante función requiere de una mayor atención por parte de la institución como centro coordinador. La orientación educativa que realizan el psicopedagogo y el maestro de apoyo se convierte en el eje del sistema de influencias para mejorar la preparación de los agentes educativos que participan en la educación de los niños sordos de la primera infancia.

Los niños con discapacidad auditiva presentan insuficiencias para comunicarse por un pobre desarrollo del lenguaje, tienen dificultades en la comprensión y por consiguiente en otros proceso cognitivos como la atención y la memoria, lo cual provoca insuficiencias en el aprendizaje.

La enseñanza de nociones elementales de matemática en la primera infancia puede contribuir al desarrollo de los procesos del pensamiento y a preparar al niño para la asimilación de contenidos matemáticos más complejos una vez que ingrese en la escuela. Esto es posible si se tiene en cuenta las particularidades del niño con discapacidad auditiva de la primera infancia.

Las sugerencias que se proponen en el presente trabajo estándirigidas a mejorar el desempeñode padres $y$ otros agentes educativos para el desarrollo de nociones elementales de matemáticas en el niño sordo

\section{DESARROLLO}

Lascaracterísticas deldesarrollohumanogeneran la riqueza y la diversidad de los seres humanos, expresada en las diferencias individuales. Entre los grandes desafíos que enfrenta hoy día la educación cubana se encuentran los que emanan de las contradicciones entre la masividad de la educación y la búsqueda de la excelencia, y entre la necesaria unidad del sistema educativo y la diversidad de personas, condiciones, y aspiraciones, de los protagonistas del proceso de enseñanza-aprendizaje (Castellanos D 2011)

Se hace imprescindible la educación inclusiva la cual se ha reconocido como una tentativa de atender la diversidad.

Según Borges S., Orosco M. (2014), la inclusión educativa encierra un conjunto de ideas que orientan la pedagogía en una dirección 
particular. En Cuba es entendida como una concepción que reconoce el derecho de todos a una educación de calidad, independientemente de sus particularidades y características que condicionan las variabilidades en su desarrollo, y que propicie la integración a la sociedad como individuos plenos en condiciones de poder disfrutar las posibilidades que ella ofrece $y$ contribuir a su perfeccionamiento.

La inclusión educativa significa garantizar desde los primeros años de vida, una educación de calidad, que implica potenciar al máximo posible el desarrollo de sus educandos y que permita alcanzar con ello los resultados deseados.

Lo anterior exigeelevar la calidad de la orientación y asesoría a la familia de niños de características muy diversas, entre ellos los niños condiscapacidad auditiva.

Castellanos R. (2013) define la discapacidad auditiva como una limitación sensorial que tiene su causa en el daño orgánico del sistema auditivo debido al cual se producen pérdidas de la audición de diferente magnitud, que dificultan la formación y desarrollo del lenguaje oral ( en su principal función: la comunicativa), y el desarrollo de los procesos psíquicos.

Investigaciones realizadas por Nieves $M$. (2000) Bravo M. (2003), Rodríguez X, (2003), Castellanos R. (2013); demuestran que los niños con discapacidad auditiva presentan insuficiencias para comunicarse por un pobre desarrollo del lenguaje, tienen dificultades en la comprensión y por consiguiente en otros proceso cognitivos como la atención y la memoria, lo cual provoca insuficiencias en el aprendizaje.

Se afirma que el lenguaje oral en el niño deficiente auditivo solo puede ser aprendido, no adquirido, ya que en este caso su asimilación no ocurre de forma natural, sin enseñanza ni organización sino todo lo contrario.
Suárez S. (2008) señala que en el niño sordo las funciones informativa, reguladora y afectiva de la comunicación se ven afectadas y añade que la adquisición temprana de la lengua de señas si se produce en etapas comparables a las que siguen los niños oyentes en su desarrollo del lenguaje permite obtener mejores resultados en su segunda lengua, aspecto que debe tenerse presente en toda la organización del currículo de la edad temprana y preescolar en la educación del niño sordo. La propia autora enfatiza en la importancia de la familia para estimular el desarrollo del lenguaje y otros procesos psíquicos a través de la lengua de señas, la dactilología y la lectura labiofacial.

La importancia de este planteamiento es que confirma la idea vigotskiana de que la discapacidad auditiva no significa otra cosa que la falta de una de las vías para la formación de los enlaces condicionados con el medio.

La primera infancia es la etapa del desarrollo que abarca desde el nacimiento hasta los 6 años. Es considerada por muchos como el período más significativo en la formación del individuo, pues en la misma se estructuran las bases fundamentales de las particularidades físicas y formaciones psicológicas de la personalidad, que se consolidarán y perfeccionarán en etapas posteriores del desarrollo.Vigotski define esta etapa como "... el período más saturado y rico en contenido, más denso y lleno de valor del desarrollo en general".

La educación temprana y prescolar de los niños con discapacidad auditiva garantiza el máximo desarrollo psicológico, a partir de una adecuada educación y considerando que es el período sensitivo para el desarrollo del lenguaje. El éxito que se obtenga en este propósito influirá positivamente en el desarrollo del pensamiento, los demás procesos cognitivos y en el desarrollo lo más integral posible de la personalidad del niño. 
La orientación educativa para el desarrollo de los niños con discapacidad auditiva de la primera infancia tiene puntos comunes con la atención temprana. Según Gerónimo O. y colectivo de autores (2015), la atención o estimulación temprana como también se conoce es considerada como un conjunto de vías que permiten potenciar el desarrollo de diferentes áreas de la actividad psíquica del menor que presenta desviaciones del desarrollo o factores de riesgo, con el fin de que alcance los índices considerados como normales, siendo el pensamiento el aspecto más trabajado. Según estos autores la labor preventiva, alcanza en la actualidad una extraordinaria importancia social, además de ser una dirección principal de trabajo del MINED y el Ministerio de la Salud para lograr mayores niveles de calidad. El niño de la primera infancia es atendido a través del Programa educa a tu hijo, círculo infantil y aulas de prescolar.

En Cuba como se señala anteriormente la atención temprana se lleva a cabo a través de la educación prescolar en cuyo programa se fundamenta y precisa que "[...] el principal objetivo es lograr el máximo desarrollo posible de cada niño -lo cual constituye premisa indispensable de su preparación para la escuela-, que de acuerdo con el fin general de nuestra educación debe ser integral y armónico [...]."(Gerónimo O. y Colectivo de autores (2015)

En el logro de este objetivo se destaca el maestro de apoyo quien se convierte en el eje del sistema de influencias para poder cumplir con la preparación de los agentes educativos que participan en la educación de los niños sordos de la primera infancia.

La función principal del maestro de apoyo es la inserción de los aspectos específicos de atención a los sordos e hipoacúsicos, al trabajo metodológico del centro integrador y la actualización del claustro en los aspectos de la especialidad.

Los educandos con discapacidad auditiva pueden vencer los objetivos de cada nivel educativo, por lo que es esencial tomar como punto de partida los logros de desarrollo de la primera infancia, los cuales se encuentran declarados en el programa de educación prescolar diferenciados según los períodos comprendidos en la primera infancia. Dicho programa se divide en cuatros ciclos para adecuar sus objetivos a las particularidades de los diferentes periodos que integran la primera infancia.

En el segundo ciclo correspondiente al segundo y tercer año de vida del niño, se inicia el trabajo con el programa de conocimiento del mundo de los objetos y sus relaciones, cuyos contenidos están encaminados a lograr que los niños al finalizar la etapa prescolar, sean capaces de descubrir, analizar y valorar las diversas cualidades presentes en los objetos del mundo que les rodea, observen las relaciones existentes entre estos y sean capaces de expresarlo.

Los conocimientos y habilidades que logra el niño en este ciclo, contribuyen a la asimilación del contenido del programa Nociones Elementales de Matemática, el cual se inicia en sexto año de vida.

En este programa se trabajan los contenidos relacionados con conjuntos y longitudes. Inicialmente se abordan los diferentes procesos de formación, reconocimiento, descomposición y unión de conjuntos, a partir de las características de los objetos. Seguidamente se inicia el trabajo con las relaciones cuantitativas mediante la comparación, lo que permite a los niños pasar al reconocimiento de las cantidades del uno al diez y operar con cantidades en procesos de formación, descomposición, unión y comparación detallada de conjuntos. 
Estos contenidos cobran especial importancia en el niño con discapacidad auditiva, ya que contribuyen al desarrollo del pensamiento y otros procesos cognitivos. Como se señaló anteriormente la discapacidad auditiva trae como consecuencias dificultades para comunicarse por el pobre desarrollo del lenguaje y esto afecta la comprensión, la atención y la memoria sobre todo en la verbal, lo cual provoca insuficiencias en el aprendizaje. Otra característica que puede apreciarse en ellos es que tienen afectadas severamente las relaciones espaciales.

La orientación educativa hacia la familia y otros educadores para la formación de las nociones elementales de matemática en el niño sordo debe realizarse teniendo en cuenta las características de la primera infancia y las particularidades de la discapacidad auditiva.

La orientación educativa para el desarrollo de nociones elementales de matemática en los niños con discapacidad auditiva de la primera infancia debe partir de los siguientes presupuestos:

- Se considera al aprendizaje como núcleo vertebrador de la intervención psicopedagógica desde la concepción de la educación inclusiva cuyo objetivo es garantizar una educación de calidad que potencia al máximo posible el desarrollo de sus educandos y que trasciende los marcos de la escuela.

- La orientación adquiere un carácter proactivo que se anticipa a la aparición de todo aquello que suponga un obstáculo al desarrollo permanente de la persona.

- Se concibe que la educación condiciona e impulsa el desarrollo, a partir de que es uno de los postulados centrales del enfoque histórico cultural.

- Se asume como punto de partida el diagnóstico explicativo que caracterice la situación social del desarrollo del niño teniendo en cuenta el desarrollo individual actual y potencial, así como las condiciones socioculturales donde vive.

- Se reconoce el papel protagónico de la familia, por constituir la primera institución social en que se logra el desarrollo físico y psicológico sobre la base de intensos lazos afectivos y emocionales.

- El psicopedagogo y la maestra de apoyo deben orientar a la familia y otros agentes educativos, para que faciliten ambientes de intercomunicación; padres-niño y niño-niño.

- Considerando que es en la actividad y la comunicación donde se desarrolla y manifiesta la personalidad, resulta recomendable, considerar la actividad fundamental en cada uno de los periodos de la infancia temprana.

- Se debe promover la comunicación e interrelación constante y estimular el desarrollo del lenguaje y otros procesos psíquicos a través de la lengua de señas, la dactilología y la lectura labiofacial como forma alternativa de comunicación.

Teniendo en cuenta lo anterior y a partir de la experiencia de la maestra de apoyo que atiende a los niños sordos de la primera infancia en la provincia Matanzas, serealizan las acciones siguientes:

I. Intercambio con los presidentes municipales para solicitar un levantamiento en cada territorio de los niños de la primera infancia, y explorar posibilidades de apoyo en cada municipio.

II. Diagnóstico inicial: Se caracterizan las familias, directivos y docentes, con el objetivo de identificar potencialidades $y$ debilidades para la atención de los niños 
de la primera infancia con discapacidad auditiva.

Se reflejan como potencialidades:

- Los especialistas, directivos, docentes y familias, muestran disposición y expresan compromiso con la tarea y satisfacción por recibir la preparación para mejorar los resultados en la labor que realizan.

- La maestra de apoyo que atiende a los niños sordos de la primera infancia en la provincia Matanzas posee más de 30 años de trabajo en esta especialidad, con una preparación sistemática y con categorías de máster en Ciencias de la Educación, Mención: Educación Especial.

\section{COMO DEBILIDADES SE SEÑALAN:}

-Insuficiente preparación de directivos y docentes para brindar atención adecuada a educandos con discapacidad auditiva y sordos con implante coclear.

-La familia no siempre cuenta con la preparación para ofrecer la ayuda que sus hijos necesitan.

III. Realización de actividades, en coordinación con la ANSOC, donde participen los educandos con discapacidad auditiva junto a sus familiares

IV. Coordinación y desarrollo de talleres de preparación grupal por zonas, para la preparación de promotores, educadoras de círculos infantiles y maestras de prescolar.

V. Visita a los hogares para preparar a las familias.

Los talleres y las visitas a los hogares se preparan a partir de las siguientes sugerencias.

Sugerencias generales para el desarrollo de nociones elementales de matemática en el niño con discapacidad auditiva.

$\checkmark$ Comprobar que domine el vocabulario necesario para la actividad. Las palabras arriba-debajo, izquierda-derecha, muchopoco, más-menos, dentro-fuera, etc., deben ser asimiladas por el niño con anterioridad a través de sistemas alternativos comunicativos(lengua de señas, gestos, dactilología, lectura labiofacial).

$\checkmark$ En caso que el niño no domine este vocabulario se utilizarán rótulos con la palabra, realizando la acción correspondiente, hasta que lo interiorice.

$\checkmark \quad$ La orientación para la tarea a realizar debe ser de manera individual, mirando a la cara para que pueda leer por lectura labiofacial y señas. Es necesario repetir la orientación más de una vez y asegurarse de que el niño ha entendido.

$\checkmark \quad$ Utilizar los niveles de ayuda que requiera el niño desde la orientación hasta la demostración del ejercicio.

$\checkmark \quad$ Se utilizarán materiales de apoyo según el caso, por ejemplo en la fundamentación de conjuntos se presentan dificultades para entenderlo o expresarlo se pueden utilizar tarjetas de apoyo con los signos +, -, =,>, <.

$\checkmark$ Tanto la familia como los educadores deben comprobar que el niño esté usando adecuadamente la prótesis auditiva o el complemento del implante coclear.

$\checkmark$ Promover actividadesvariadas y atractivaspara los niños, que les propicie el desarrollo de su creatividad, enriquecimiento personal, estado emocional positivo y ampliar sus vivencias.

$\checkmark$ De dos a tres años de vida, la actividad estará encaminada a realizar acciones de 
correlación con instrumentos, materiales, juguetes y otros objetos para "descubrir" su función, su nombre, sus características.

$\checkmark$ De tres a seis años el juego, debe favorecer la asimilación de representaciones sobre las diversas propiedades y relaciones entre los objetos a partir de su forma, color y tamaño.

A continuación se ilustra cómo se orienta a los padres para realizar actividades de conocimiento del mundo de los objetos y nociones de matemáticas.

Actividad No 1. Conocimiento del mundo de los objetos.

Tema:Juego y aprendo con los objetos.

Objetivo: Preparar a la familia para el trabajo con las cualidades de los objetos: color, forma y tamaño.

Desarrollo: Se inicia preguntando a la familia con un intercambio sobre sus experiencias, logros y dificultades en la educación de sus hijos.

Se invita a los participantes a formar el mensaje a partir de los rótulos dados y expresar sus criterios. Los rótulos son los siguientes:

(INDEPENDENCIA), (Y APOYO), (NO SE EXCLUYEN), (AMBOS SON NECESARIOS), (PARA ACTUAR DE FORMA INTELIGENTE)

Se debate y se recomienda a partir del análisis del mensaje cómo aprovechar las situaciones que se producen cotidianamente en el hogar para enseñar las cualidades de los objetos: color, forma y tamaño, entre otras actividades.

Se analizan algunas actividades que aparecen en el Folleto "Educa a tu Hijo", para que posteriormente sean aplicadas en los hogares con las adaptaciones pertinentes para el niño sordo.
A modo de ejemplo se le explica cómo realizar las siguientes actividades:

Se les explica que todas tienen como objetivo conocer y diferenciar el color, la forma, el tamaño de los objetos.

\section{ACTIVIDADES:}

1. Pídale a su niño que de un conjunto de juguetes y objetos diferentes, agrupe los que sean de un mismo color y luego pregúntele de qué color es. Cuando ya sepa agrupar objetos por sus color, dirija su atención a que algunos son más claros y otros más oscuros. Enséñelo a que compare, por ejemplo las hojitas, y que ponga a un lado todas las que son verde oscuro y al otro, las verdes claro. Puede hacer lo mismo con florecitas, carretes de hilo, lápices de colores, pedacitos de papel de colores y otros. Verá cómo le gusta esta actividad.

2. Ya su pequeño sabe agrupar objetos y juguetes que tiene una forma igual. Ahora, ya puede mostrar un círculo, por ejemplo y pedirle que busque a su alrededor objetos que tengan una forma parecida: un reloj, un cenicero, una pelota, un platico. Haga lo mismo con el cuadrado, el rectángulo, el óvalo y el triángulo. Así aprende mejor a conocer la variedad de formas que hay en el mundo que lo rodea.

3. Juegue con él a colocar, por tamaño, los palitos, piedrecitas, hojitas, florecitas que hayan recogido en los paseos que dan junto. Pídale que lo ordene de mayor a menor, sino puede hacerlo a simple vista, enséñelo a que los compare colocándolo uno al lado del otro.

4. Invítelo también a que ponga en fila, por orden de tamaño, cuatro muñecas, carritos o pelotas (puede ser de papel); cuando lo haya hecho, ofrézcale una más pequeñita o una más grande para que la coloque al 
final y al principio, según corresponda, $\mathrm{Si}$ no sabe hacerlo, enséñelo a comparar y a determinar su lugar.

5. Enséñele las posiciones que ocupan los objetos en el espacio. Esto también es muy importante para que aprenda a orientarse. Juegue con su niño a ¿y ahora dónde está? Coloque un muñequito $u$ otro juguete delante, detrás o a un lado de él y pídale que le diga dónde está. Luego dígale que cierre los ojos, cambie de lugar el objeto y pregúntele: ¿y ahora dónde está? Verá que el muñequito cambió de lugar con respecto a él; esto le resultará muy divertido.

Evaluación: ¿Demuestre con un ejemplo, en dependencia de la edad de su niño cómo le puede enseñar la cualidad color (forma, tamaño) de los objeto?

Para concluir se invita a ejercitar el vocabulario en lengua de señas correspondiente (color, mayor, menor, delante, detrás)

Actividad No 2. Nociones elementales de matemática.

Tema: Cuántos hay y cuanto hay

Objetivo: Preparar a la familia para el trabajo con las cualidades de los objetos: cantidad y medida.

Ya su niño conoce las cualidades de los objetos como forma, color, tamaño y lo relaciona; lo que son más bonitos, los más pequeños, lo que están arriba y abajo. Cuando lo ayuda a que lo relacione por su cantidad también lo prepara para que le sea fácil aprender matemática en la escuela.

\section{LE SUGERIMOS ACTIVIDADES COMO ESTAS:}

1. Observe que los objetos pueden ser diferentes, pero la cantidad es la misma. ¿Dónde hay más? ¿Dónde hay menos?
Guiándose por estos ejercicios, usted puede hacerle otras preguntas basadas en todas las cosas que rodean al niño.

Cuando las cantidades son pequeñas, el niño puede resolverlas directamente a simple vista; otras veces necesita, por ejemplo, piedrecilla, semillitas.

2. Ya con esos conocimientos puede jugar con su papá al dominó de seis. Si no tienen, pueden construirlo con tarjetas de cartón.

Aprender a medir es algo muy importante. El niño debe aprender que se puede medir muchas cosas y que los instrumentos para medir puede ser muy variados.

3. Juegue a medir el largo y el ancho de una mesa, del cuarto, de los surcos sembrados. Para medirlo puede utilizar palitos, cordeles $\mathrm{u}$ otras cosas que tengan a mano. Si lo que van a medir es largo, pueden hacer marcas y poner ejemplo, una piedrecita en cada marca. La cantidad de piedrecitas les ayudará a comparar.

\section{- ¿Cuál es más larga?}

\section{- ¿Cuál es más alta?}

4. Su niño debe saber que los líquidos se miden. Juega con él a descubrir en qué cubo hay más agua ¿Qué medida puedes utilizar? Puede ser un vaso, un jarrito, un cucharón. Depende de la cantidad de líquido que mida. Si coloca una piedrecita por cada jarrito de agua que saque, verá qué fácil resulta este juego para su niño.

Y aquí ¿Quién recogió más frijoles? La medida puede ser ahora una latica. El propio granito de frijol puede marcar el número de laticas recogida.

5. Aunque aún parece pequeño, ya su niño puede aprender a resolver problemas:

- Dos amiguitos están jugando a las bolas, 
uno tiene tres y el otro también tiene tres ¿Cuántas bolas hay?

- En el árbol hay cinco pajaritos y se van volando dos ¿Cuántos se quedaron en el árbol?

- Papá trajo una cestica con mamoncillos y quiere que sus tres niños cojan la misma cantidad. ¿Qué hacer?

Para los niños de esta edad los problemas deben estar dibujados. Usted puede hacerle esos sencillos dibujos. Ellos los resuelven mejor utilizando los mismos objetos, fichitas o semillitas.

Seguro que usted puede preparar muchas tareas y materiales para jugar con su niño. Una vez que su niño ingrese en la escuela la maestra de primer grado y su propio hijo le agradecerá mucho su dedicación.

Se les insiste a los padres que además de los objetos recomendados deben preparar algunos materiales de apoyo como rótulos con palabras y figuras geométricas para la realización de estas actividades.

\section{CONCLUSIONES}

- El programa de conocimiento del mundo de los objetos y sus relaciones, así como el de nociones elementales de matemática son de gran importancia en la educación del niño sordo, ya que contribuyen al desarrollo del pensamiento y otros procesos cognitivos y lo prepara para su ingreso a la escuela.

- En el diagnóstico inicial realizado se constató que existen potencialidades y dificultades en los diferentes contextos, para el desarrollo de las nociones elementales de matemática en el niño con discapacidad auditiva, hipoacúsicos y sordos con implante coclear.

- Posterior a la aplicación del sistema de acciones se logran resultados positivos, que de manera general se enmarcan en una mejor preparación tanto de los directivos, docentes, familia, para el desarrollo de nociones elementales de matemática en el niño sordo.

\section{REFERENCIAS BIBLIOGRÁFICAS}

Bell Rodríguez, R. \& López Machín R. (2000). Convocados por la diversidad. La Habana: Ed. Pueblo y Educación.

Borges Rodríguez, S. \& Orozco Delgado M. (2014). Inclusión Educativa y Educación Especial: Un horizonte singular y diverso para igualar las oportunidades de desarrollo. La Habana: Educación Cubana.

Castellanos, R. M., \& Xiomara, R. (2013). Sordera. Aspectos Psicológicos. La Habana: Pueblo y Educación.

Cobas Ochoa, C. L. \& Gayle Morejón A. (2010). Atención a la diversidad del alumnado en el contexto escolar. Formato digital.

Chkout, T., Orozco, M. \& Borges, S. (2013). Sobre el perfeccionamiento de la Educación Especial. La Habana: Ed. Pueblo y Educación.

Educación para todos: Todas las posibilidades son aceptadas. (2010). (Consultado marzo del 2015). Recuperado de www.lainsignia.org

Farñas, G. (2003). Vigostky en la Educación Superior Contemporánea. Habana, Cuba. Recuperado el 15 de 3 de 17

Fernández, G., \& colectivo de autores. (2009). Prevención y atención al niño de 0 a 6 años con necesidades educativas especiales. Pedagogía. Curso 28. Habana, Cuba. Recuperado el 11 de 2 de 18

Gerónimo, O. (2015). Una propuesta para la atención integral a niñas/os con 
necesidades educativas especiales de 0-6 años en la provincia Sancti- Spíritus. Pedagogía 2015 Encuentro por la unidad de los educadores Sancti Spíritus ISBN 978-958-18-1024-3. Recuperado el 11 de 10 de 2017, de Pedagogía 2015 Encuentro por la Unidad de los Educadores. Sancti Spíritus .

López Machín, R. (2011). Diversidad e igualdad de oportunidades en la escuela. Selección de temas para los docentes. La Habana: Ed. Pueblo y Educación.

Nieves, M. (Julio-Agosto de 2000). Aproximación a los conceptos de necesidad educativa y necesidades educativas especiales. (I. C. Pedagógicas, Ed.) Desafío Escolar. Revista Iberoamericana de Pedagogía, 49-52.

ONU. CEPAL. (2016). Agenda 2030 y los Objetivos de Desarrollo Sostenible. Una oportunidad para América Latina y el Caribe. Consultado en página web de las Naciones Unidas www.un.org/ sustainabledevelopment/es

Ríos Moreno, D. (2008). La orientación profesional en adolescentes sordos, en condiciones de inclusión, en la provincia Matanzas. Tesis presentada en opción al título de Dr. C. Universidad Camilo Cienfuegos, Matanzas. Cuba.

Rodríguez Fleitas, X. (2005). Proyecto Educativo. Modelo Cubano de Educación Bilingüe para Personas Sordas. En III Congreso Regional de Psicología. La Habana, Cuba.

Rodríguez Fleitas, X. (2007). Una mirada Reflexiva hacia el niño sordo. La Habana: Ed. Pueblo y Educación.

Suárez Deán, Susana. (2008). La educación familiar para la comunicación bilingüe con el niño sordo de edad temprana y preescolar. Tesis de Maestría en Ciencias de la Educación. ISP." Juan Marinello", Matanzas. Cuba.

Torres Ramos, Y. (2018) El empleo de los medios del proceso educativo para el desarrollo integral del niño en la primera infancia. Revista REDIE, Revista electrónica de la Red Durango de investigadores educativos. México.

UNICEF. (2005). Inclusión de niños con discapacidad en la escuela regular. Hacia el desarrollo de escuelas inclusivas. Fundación HINENI.

Vigotski, L. S. (1995) Obras Completas. Tomo V. Fundamentos de Defectología. La Habana: Ed. Pueblo y Educación. 